\title{
ENTIRE FUNCTIONS OF SINE TYPE AND THEIR APPLICATIONS
}

\author{
R. A. BASHMAKOV, A. A. PUTINTSEVA, AND P. C. YULMUKHAMETOV
}

\begin{abstract}
For subharmonic functions that depend only on the real part of $z$, new constructions of "sine type functions" are presented. This term is reserved for entire functions whose deviation from a given function is majorized, everywhere except some collection of disks, by a certain constant. It is shown that the system of exponentials constructed by the zeros of a sine type function for some convex function is complete and minimal in a certain weighted Hilbert space on an interval of the real line.
\end{abstract}

\section{INTRODUCTION}

In this paper, we construct entire functions asymptotically close to $u(\operatorname{Re} z)$, where $u$ is a convex function on the real line prescribed beforehand. The results are applied to the study of systems of exponentials $\exp \left(\lambda_{k} t\right)$ (where the $\lambda_{k}, k=1,2, \ldots$, are the zeros of an entire function) in weighted Hilbert spaces on intervals of the real line.

Definition. Let $u$ be a real subharmonic function on the plane, and let $\tau(u, z)$ be the radius of the largest disk centered at $z$ such that $u$ deviates from the space of harmonic functions on this disk by at most 1 :

$$
\begin{array}{r}
\tau(u, z)=\sup \{r>0: h(w) \text { is harmonic in the disk } B(z, r) \\
\text { and } \left.\max _{w \in B(z, r)}|h(w)-u(w)| \leq 1\right\} .
\end{array}
$$

By a sign type function for $u$ we mean an entire function $L$ satisfying the following conditions.

1. All zeros $z_{n}, n \in \mathbb{N}$, of $L$ are simple, and for some $\varepsilon>0$ the disks $B\left(z_{n}, \varepsilon \tau\left(u, z_{n}\right)\right)$, $n \in \mathbb{N}$, are pairwise disjoint.

2. For every $\varepsilon>0$, outside the union of the disks $B\left(z_{n}, \varepsilon \tau\left(u, z_{n}\right)\right), n \in \mathbb{N}$, we have

$$
|\ln | L(z)|-u(z)| \leq A(\varepsilon) .
$$

By subharmonicity and the definition of $\tau(u, z)$, we also obtain the following property. $2^{\prime}$. For $z \in \mathbb{C}$, we have the upper estimate

$$
\ln |L(z)| \leq|u(z)|+A_{1}(\varepsilon) .
$$

Apparently, the term "a function of sine type" first appeared in [9]. In terms of the definition in the present paper, this was a function of sine type of the function $h(z)=\pi|\operatorname{Re} z|$. Then, in [1, functions of sine type for $h(z)=\max \{w \in D: \operatorname{Re} z w\}$ were used ( $D$ is a bounded convex polygon).

Several years before the paper [1] appeared, Azarin had proved a theorem (see [2]) on approximation of an arbitrary subharmonic function of finite order by the logarithm

2010 Mathematics Subject Classification. Primary 30D15.

Key words and phrases. Entire functions, Hilbert spaces, completeness and minimality for a system of exponentials, Fourier-Laplace transformation.

Supported by RFBR (grants nos. 08-01-97020-p_Volga_a, 10-01-00233-a). 
of the modulus of an entire function. Azarin's result was refined substantially in 3 . Among other things, in that paper it was proved that for every subharmonic function $u$ of finite order there exists an entire function $f$ such that

$$
|u(z)-\ln | f(z)||=O(\ln |z|), \quad z \notin E,|z| \longrightarrow \infty,
$$

outside of an exceptional set $E$ of finite measure. It can easily be shown that the sine type functions introduced above share the properties of entire functions constructed in [3.

In [4], sine type functions were constructed for support functions of convex domains with boundary whose curvature is bounded and bounded away from zero.

In [7, subharmonic functions of slow growth were studied.

A special tribute should be paid to the paper [10, which is the most substantial among the studies on approximation of subharmonic functions over recent years. As far as the exactness of the asymptotics is concerned, the results of that paper resemble the results about sine type functions. However, for sine type functions, the construction of the exceptional set is at least of the same (if not of greater) importance as the sharpness of estimates. In this respect, Theorem 3 in [10] seems promising, because it contains a transparent description of the exceptional set. However, that description involves the zero set of unclear structure for the entire function in question. It may happen that fine methods of [10] would provide sine type functions in the setting of the present paper. The emphasis of the latter is on the fact that our approach makes it possible, though in a rather particular case, to do without complicated theorems (such as the theorem on measure decomposition in [3]) and without "annihilation of moments". As a result, the construction of sine type functions turns out to be quite transparent. Concerning the fact that we treat only quite particular entire functions that only depend on $\operatorname{Re} z$, it should be noted that the sine type functions constructed here suffice to investigate completeness, minimality, and the basis property for systems of exponentials and reproducing kernels in a broad class of Hilbert function spaces on the real line.

We denote by $\mathcal{S}(u)$ the set of sine type functions for a function $u$.

\section{§1. Construction of Sine type functions}

Our constructions will be based on the function $F(z)=e^{z}-1$, so we begin with a description of its properties. We put $\operatorname{Re}^{+} z=\max (0, \operatorname{Re} z)$.

$F 1$. The zeros of $F$ are the points $z_{n}=2 \pi i n, n \in \mathbb{Z}$; all zeros are simple.

$F 2$. For every $\varepsilon>0$, outside the union of the disks $B\left(z_{n}, \varepsilon\right), n \in \mathbb{Z}$, we have

$$
|\ln | F(z)\left|-\operatorname{Re}^{+} z\right| \leq A_{\varepsilon} e^{-|\operatorname{Re} z|},
$$

where $A_{\varepsilon}$ is a constant.

These properties can easily be deduced from the estimates of $\sin z$ (see, e.g., [5, p. 52]), because the Euler formula implies the identity

$$
F(z)=2 i e^{\frac{z}{2}} \sin \left(-\frac{i z}{2}\right) .
$$

Let $L(x)=d(x-b)$ be a real linear function, and let $L^{+}(x)=\max (0, L(x))$. The following properties are direct consequences of $F 1$ and $F 2$.

$L F 1$. The zeros of $F(L(z))$ are the points $z_{n}=b+\frac{2 \pi i n}{d}, n \in \mathbb{Z}$, and all zeros are simple.

$L F 2$. For every $\varepsilon>0$, outside the union of the disks $B\left(z_{n}, \frac{\varepsilon}{d}\right), n \in \mathbb{Z}$, we have

$$
|\ln | F(L(z))\left|-L^{+}(\operatorname{Re} z)\right| \leq A_{\varepsilon} e^{-|L(\operatorname{Re} z)|} .
$$

We prove two preparatory lemmas. 
Lemma 1. Let $u$ be a piecewise linear convex function on $\mathbb{R}$ such that

$$
u(x)=l_{k}(x), \quad x \in\left[b_{k} ; b_{k+1}\right], \quad k \in \mathbb{Z},
$$

where $l_{k}$ is a linear function and $b_{k}$ is a monotone increasing sequence of break points. Putting $L_{0}(x)=l_{0}(x), L_{n}(x)=l_{n}(x)-l_{n-1}(x), n \neq 0$, we have

$$
u(x)=L_{0}(x)+\sum_{n \neq 0} L_{n}^{+}(x), \quad x \in \mathbb{R} .
$$

Proof. This is fairly simple.

The following geometric characteristic of convex functions was introduced in [6]. Let $u$ be a convex function on the real line; for every $y \in \mathbb{R}$ we denote by $\rho(u, y)$ the supremum of all $p>0$ such that

$$
\int_{-p}^{+p}\left|u_{+}^{\prime}(y+t)-u_{+}^{\prime}(y)\right| d t \leq 1 .
$$

If $u$ is nonlinear, then $\rho(u, y)$ is a finite function on $\mathbb{R}$ and, as was shown in the same paper [6], it satisfies the Lipschitz condition

$$
|\rho(u, x)-\rho(u, y)|<|x-y|, \quad x \neq y .
$$

The definition of $\rho(u, y)$ can also be given in the following form: this is the supremum of all $p>0$ such that

$$
u(y+p)+u(y-p)-2 u(y) \leq 1 .
$$

For $y \in \mathbb{R}$, we denote by $l_{u}(y, x)$ the linear function equal to $u(x)$ at $y \pm \rho(u, y)$. Geometric considerations easily yield the inequality

$$
0 \leq l_{u}(y, x)-u(x) \leq 1, \quad x \in[y-\rho(u, y) ; y+\rho(u, y)] .
$$

Lemma 2. For every convex function $u$ on $\mathbb{R}$, there exists a piecewise linear convex function $v$ such that

1) if $b_{n}, n \in \mathbb{Z}, \cdots<b_{-1}<b_{0}<b_{1}<\cdots$ are break points for $v$ and $b_{n}^{*}=\left(b_{n}+b_{n+1}\right) / 2$, then

$$
b_{n+1}-b_{n}=2 \rho\left(u, b_{n}^{*}\right), \quad n \in \mathbb{Z}
$$

moreover, if $u$ has an asymptote at $+\infty($ at $-\infty)$, then the sequence $b_{n}$ is finite from the right (from the left);

2) for all $x \in \mathbb{R}$ we have

$$
u(x) \leq v(x) \leq u(x)+1 ;
$$

3) if

$$
v(x)=l_{n}(x), \quad x \in\left[b_{n} ; b_{n+1}\right], \quad n \in \mathbb{Z},
$$

and $d_{n}$ is the slope of the linear function $l_{n}-l_{n-1}$, then

$$
\frac{1}{2 \rho\left(u, b_{n}^{*}\right)} \leq d_{n} \leq \frac{3}{\rho\left(u, b_{n}\right)}, \quad n \in \mathbb{Z}
$$

Proof. Taking $b_{0}^{*} \in \mathbb{R}$ arbitrarily, we put

$$
b_{0}=b_{0}^{*}-\rho\left(u, b_{0}^{*}\right), \quad b_{1}=b_{0}^{*}+\rho\left(u, b_{0}^{*}\right), \quad v(x)=l_{u}\left(b_{0}^{*}, x\right), \quad x \in\left[b_{0} ; b_{1}\right] .
$$

By (2), we have

$$
u(x) \leq v(x) \leq u(x)+1, \quad x \in\left[b_{0} ; b_{1}\right] .
$$

By (1), the functions $x \pm \rho(u, x)$ are monotone increasing. Thus, the equation

$$
x-\rho(u, x)=b_{1}
$$

can have at most one solution. If it exists, we denote it by $b_{1}^{*}$ and put

$$
b_{2}=b_{1}^{*}+\rho\left(u, b_{1}^{*}\right), \quad v(x)=l_{u}\left(b_{1}^{*}, x\right), \quad x \in\left[b_{1} ; b_{2}\right] .
$$


If there is no solution, the function $u$ has an asymptote at $+\infty$, that is,

$$
\lim _{x \rightarrow+\infty}(u(x)-(k x+c))=0
$$

for some linear function $k x+c$. In this case, we take the rightmost break point for $v$ for the role of $b_{1}$ and put $v(x)=k\left(x-b_{1}\right)+u\left(b_{1}\right)$ for $x>b_{1}$. Again, (2) implies that

$$
u(x) \leq v(x) \leq u(x)+1, \quad x \geq b_{1} .
$$

If the equation $x+\rho(u, x)=b_{0}$ has a solution, we denote it by $b_{-1}^{*}$ and put

$$
b_{-1}=b_{-1}^{*}-\rho\left(u, b_{-1}^{*}\right), \quad v(x)=l_{u}\left(b_{-1}^{*}, x\right), \quad x \in\left[b_{-1} ; b_{0}\right] .
$$

If it has none, we let $b_{0}$ be the leftmost break point for $v$, and we put $v(x)=k\left(x-b_{0}\right)+$ $u\left(b_{0}\right)$ for $x<b_{0}$, where $k=\lim _{x \rightarrow-\infty} u^{\prime}(x)$. Continuing in this way as far as necessary, we construct a function $v(x)$ that obviously possesses properties 1) and 2).

We verify the third property. By construction, we have

$$
v(x)=l_{n}(x)=l_{u}\left(b_{n}^{*}, x\right), \quad x \in\left[b_{n} ; b_{n+1}\right] ;
$$

consequently, $l_{n-1}(x) \leq u(x)$ on $\left[b_{n} ; b_{n+1}\right]$. Therefore,

$$
l_{n}\left(b_{n+1}\right)-l_{n-1}\left(b_{n+1}\right)=2\left(l_{n}\left(b_{n}^{*}\right)-l_{n-1}\left(b_{n}^{*}\right)\right) \geq 2\left(l_{n}\left(b_{n}^{*}\right)-u\left(b_{n}^{*}\right)\right)=1 .
$$

Since $b_{n+1}-b_{n}=2 \rho\left(u, b_{n}^{*}\right)$ and $l_{n}\left(b_{n}\right)=l_{n-1}\left(b_{n}\right)$, we obtain

$$
d_{n}=\frac{l_{n}\left(b_{n+1}\right)-l_{n-1}\left(b_{n+1}\right)}{b_{n+1}-b_{n}} \geq \frac{1}{2 \rho\left(u, b_{n}^{*}\right)} .
$$

This proves the lower estimate in 3).

We pass to the upper estimate. By (1), we have

$$
\rho\left(u, b_{n}\right)<\left|b_{n}-b_{n}^{*}\right|+\rho\left(u, b_{n}^{*}\right)=2 \rho\left(u, b_{n}^{*}\right)
$$

therefore

$$
b_{n}+\rho\left(u, b_{n}\right)<b_{n}+2 \rho\left(u, b_{n}^{*}\right)=b_{n+1} .
$$

Similarly,

$$
\rho\left(u, b_{n}\right)<\left|b_{n}-b_{n-1}^{*}\right|+\rho\left(u, b_{n-1}^{*}\right)=2 \rho\left(u, b_{n-1}^{*}\right)
$$

and $b_{n}-\rho\left(u, b_{n}\right)>b_{n-1}$. Put $\rho=\rho\left(u, b_{n}\right)$. By (2), we have

$$
\begin{aligned}
l_{n}\left(b_{n}+\rho\right) & =l_{u}\left(b_{n}^{*}, b_{n}+\rho\right) \leq u\left(b_{n}+\rho\right)+1, \\
l_{n-1}\left(b_{n}-\rho\right) & =l_{u}\left(b_{n-1}^{*}, b_{n}-\rho\right) \leq u\left(b_{n}-\rho\right)+1,
\end{aligned}
$$

whence

$$
l_{n}\left(b_{n}+\rho\right)+l_{n-1}\left(b_{n}-\rho\right)-2 u\left(b_{n}\right) \leq u\left(b_{n}+\rho\right)+u\left(b_{n}-\rho\right)-2 u\left(b_{n}\right)+2 \leq 3 .
$$

Let $l_{n}(x)=a_{n}\left(x-b_{n}\right)+u\left(b_{n}\right)$ and $l_{n-1}(x)=a_{n-1}\left(x-b_{n}\right)+u\left(b_{n}\right)$. Then the last inequality means that

On the other hand,

$$
a_{n}-a_{n-1} \leq \frac{3}{\rho}=\frac{3}{\rho\left(u, b_{n}\right)}
$$

$$
d_{n}=\frac{l_{n}\left(b_{n+1}\right)-l_{n-1}\left(b_{n+1}\right)}{b_{n+1}-b_{n}}=a_{n}-a_{n-1} \leq \frac{3}{\rho\left(u, b_{n}\right)} .
$$

This completes the proof of Lemma 2.

Now we are in a position to state and prove the main theorem. 
Theorem 1. Let $u$ be a convex function on $\mathbb{R}$. Suppose that there exists a function $\alpha(x) \geq 1$ satisfying the following conditions.

(a) There exists a constant $A$ such that for every $x \in \mathbb{R}$ and $y \in[x-\rho(u, x) ; x+\rho(u, x)]$ we have

$$
|\alpha(y)-\alpha(x)| \leq A
$$

(b) The following integral converges:

$$
\int_{-\infty}^{+\infty} e^{-\alpha(x)} \frac{d x}{\rho(u, x)}<\infty .
$$

(c) There is $a>0$ such that for every $x \in \mathbb{R}$ and $y_{1}, y_{2} \in[x-2 \alpha(x) \rho(u, x) ; x+$ $2 \alpha(x) \rho(u, x)]$ we have

$$
\frac{\rho\left(u, y_{1}\right)}{\rho\left(u, y_{2}\right)} \geq a
$$

Then there exists an entire function $f$ that has simple zeros of the form $z_{n k}=b_{n}+\frac{2 \pi i}{d_{n}} k$, $n, k \in \mathbb{Z}$, and for $\varepsilon>0$ satisfies

$$
|\ln | f(z)|-u(z)| \leq A(\varepsilon)
$$

outside the disks $B\left(z_{n k}, \varepsilon \rho\left(u, b_{n}\right)\right)$. Moreover, if $\varepsilon<\frac{1}{2}$, the disks $B\left(z_{n k}, \varepsilon \rho\left(u, b_{n}\right)\right)$ are mutually disjoint.

Proof. We describe the construction of $f$.

By Lemma 2, starting with $u$ we construct a convex piecewise linear function $v$ with breaks at $b_{n}, n \in \mathbb{Z}$, that satisfies the inequalities

$$
u(x) \leq v(x) \leq u(x)+1, \quad x \in \mathbb{R} .
$$

By Lemma 1, $v$ can be represented in the form

$$
v(x)=L_{0}(x)+\sum_{n \neq 0} L_{n}^{+}(x),
$$

where the $L_{n}$ are linear functions and, if $L_{n}(x)=d_{n}\left(x-b_{n}\right)$, statement 3) in Lemma 2 shows that

$$
\frac{1}{2 \rho\left(u, b_{n}^{*}\right)} \leq d_{n} \leq \frac{3}{\rho\left(u, b_{n}\right)}
$$

We put $F(z)=e^{z}-1$ and

$$
f(z)=e^{L_{0}(z)} \prod_{n \neq 0} F\left(L_{n}(z)\right)
$$

Then $f$ has simple zeros at the points $z_{n k}=b_{n}+\frac{2 \pi i k}{d_{n}}$, and

$$
|\ln | f(z)|-u(\operatorname{Re} z)| \leq|\ln | f(z)|-v(\operatorname{Re} z)|+1 \leq \sum_{n \neq 0}|\ln | F\left(L_{n}(z)\right)\left|-L_{n}^{+}(\operatorname{Re} z)\right|+1 .
$$

By properties $L F 1$ and $L F 2$, for every $\varepsilon>0$, outside of the disks $B\left(z_{n k}, \frac{\varepsilon}{d_{n}}\right)$ we have

$$
|\ln | f(z)|-u(\operatorname{Re} z)| \leq A(\varepsilon) \sum_{n \neq 0} e^{-\left|L_{n}(\operatorname{Re} z)\right|}+1 .
$$

We estimate the sum on the right. By the left inequality in (5), we obtain

$$
e^{-\left|L_{n}(x)\right|} \leq \exp \left(-\frac{\left|x-b_{n}\right|}{2 \rho\left(u, b_{n}^{*}\right)}\right) .
$$


Fixing $x \in \mathbb{R}$, we split all indices $n \in \mathbb{Z}$ into two portions as follows:

$$
\begin{aligned}
& Z_{1}=\left\{n \in \mathbb{Z},\left|x-b_{n}\right| \geq\left(2 \alpha\left(b_{n}^{*}\right)-1\right) \rho\left(u, b_{n}^{*}\right)\right\}, \\
& Z_{2}=\left\{n \in \mathbb{Z},\left|x-b_{n}\right|<\left(2 \alpha\left(b_{n}^{*}\right)-1\right) \rho\left(u, b_{n}^{*}\right)\right\} .
\end{aligned}
$$

Then (7) implies that

$$
\sum_{n \in Z_{1}} e^{-\left|L_{n}(x)\right|} \leq \sqrt{e} \sum_{n \in Z_{1}} e^{-\alpha\left(b_{n}^{*}\right)}
$$

By assumption, we have

$$
\sum_{n=-\infty}^{\infty} e^{-\alpha\left(b_{n}^{*}\right)} \leq \sum_{n=-\infty}^{\infty} \frac{2 e^{A}}{a} \int_{b_{n}}^{b_{n+1}} e^{-\alpha(x)} \frac{d x}{\rho(u, x)}=\frac{2 e^{A}}{a} \int_{-\infty}^{+\infty} e^{-\alpha(x)} \frac{d x}{\rho(u, x)}=C_{1}<\infty .
$$

Thus,

$$
\sum_{n \in Z_{1}} e^{-\left|L_{n}(x)\right|} \leq \sqrt{e} C_{1}
$$

Consider the indices in $Z_{2}$. Put

$$
N_{1}=\inf \left\{p: p \in Z_{2}\right\}, N_{2}=\sup \left\{p: p \in Z_{2}\right\}, s=\min \left\{p: p \in Z_{2}, b_{p} \geq x\right\} .
$$

If $n \in Z_{2}$ and $n \geq s$, then

$$
\left|x-b_{n}\right|=\left(b_{s}-x\right)+\sum_{p=s}^{n-1}\left(b_{p+1}-b_{p}\right) \geq 2 \sum_{p=s}^{n-1} \rho\left(u, b_{p}^{*}\right) .
$$

Let $p=s, \ldots, n-1$. Then

$$
b_{p} \geq b_{s} \geq x \geq b_{n}-\left(2 \alpha\left(b_{n}^{*}\right)-1\right) \rho\left(u, b_{n}^{*}\right)=b_{n}^{*}-2 \alpha\left(b_{n}^{*}\right) \rho\left(u, b_{n}^{*}\right)
$$

and, on the other hand, $b_{p} \leq b_{n} \leq b_{n+1}=b_{n}^{*}+\rho\left(u, b_{n}^{*}\right)$. Consequently, we have $b_{p} \in$ $\left[b_{n}^{*}-2 \alpha\left(b_{n}^{*}\right) \rho\left(u, b_{n}^{*}\right) ; b_{n}^{*}+2 \alpha\left(b_{n}^{*}\right) \rho\left(u, b_{n}^{*}\right)\right]$, and condition c) shows that

$$
\rho\left(u, b_{p}^{*}\right) \geq a \rho\left(u, b_{n}^{*}\right) .
$$

Together with (9), this yields

$$
\left|x-b_{n}\right| \geq 2 a \sum_{p=s}^{n-1} \rho\left(u, b_{n}^{*}\right)=2 a(n-s) \rho\left(u, b_{n}^{*}\right) .
$$

But if $n \in Z_{2}$ and $n \leq s-1$, in a similar manner we obtain

$$
\left|x-b_{n}\right|=\left(x-b_{s-1}\right)+\sum_{p=n+1}^{s-1}\left(b_{p}-b_{p-1}\right) \geq 2 a(s-n-1) \rho\left(u, b_{n}^{*}\right) .
$$

Combining these two estimates, we arrive at

$$
\frac{-\left|x-b_{n}\right|}{\rho\left(u, b_{n}^{*}\right)} \leq-2 a(|s-n|-1)
$$

By (7), it follows that

$$
\sum_{n \in Z_{2}} e^{-\left|L_{n}(\operatorname{Re} z)\right|} \leq e^{a} \sum_{k=0}^{\infty} e^{-a k} \leq \frac{e^{2 a}}{e^{a}-1} .
$$

Recalling (6) and (8), we deduce that

$$
|\ln | f(z)|-u(\operatorname{Re} z)| \leq 1+A(\varepsilon)\left(\sqrt{e} C_{1}+\frac{e^{2 a}}{e^{a}-1}\right)
$$

outside the disks $B\left(z_{n k}, \frac{\varepsilon}{d_{n}}\right)$, for every $\varepsilon>0$. By (5), this estimate holds true outside the disks $B\left(z_{n k}, \varepsilon \rho\left(u, b_{n}^{*}\right)\right.$. Since $b_{n} \in\left[b_{n}^{*}-\rho\left(u, b_{n}^{*}\right) ; b_{n}^{*}+\rho\left(u, b_{n}^{*}\right)\right]$, condition c) implies the 
inequality $\rho\left(u, b_{n}\right) \geq a \rho\left(u, b_{n}^{*}\right)$; consequently, the estimate in question holds true outside the disks $B\left(z_{n k}, \varepsilon \rho\left(u, b_{n}\right)\right)$.

It remains to prove that, for $\varepsilon<\frac{1}{2}$, the disks $B_{n k}(\varepsilon)=B\left(z_{n k}, \varepsilon \rho\left(u, b_{n}\right)\right)$ are mutually disjoint. Take two different zeros $z_{n k}, z_{m p}$. If $n=m$, then $k \neq p$ and, by statement 3 ) in Lemma 2, we have

$$
\left|z_{n k}-z_{m p}\right| \geq \frac{2 \pi}{d_{n}} \geq \frac{2 \pi}{3} \rho\left(u, b_{n}\right)>2 \varepsilon \rho\left(u, b_{n}\right),
$$

so that the disks $B_{n k}(\varepsilon)$ and $B_{m p}(\varepsilon)$ do not intersect. Suppose $n \neq m(m<n$ for definiteness). From (4) we obtain

$$
\left|z_{n k}-z_{m p}\right| \geq b_{n}-b_{m} \geq b_{n}-b_{n-1}=2 \rho\left(u, b_{n-1}^{*}\right) \geq \rho\left(u, b_{n}\right)
$$

and (3) shows that

$$
\left|z_{n k}-z_{m p}\right| \geq b_{n}-b_{m} \geq b_{m+1}-b_{m}=2 \rho\left(u, b_{m}^{*}\right) \geq \rho\left(u, b_{m}\right) .
$$

Thus,

$$
\left|z_{n k}-z_{m p}\right| \geq \frac{1}{2}\left(\rho\left(u, b_{n}\right)+\rho\left(u, b_{m}\right)\right)>\varepsilon\left(\rho\left(u, b_{n}\right)+\rho\left(u, b_{m}\right)\right) .
$$

So, the disks $B_{n k}(\varepsilon)$ and $B_{m p}(\varepsilon)$ do not intersect.

Corollary 1. If u satisfies the assumptions of Theorem 1, then there exists a sine type function for it.

Proof. We show that the function $f$ constructed in Theorem 1 is a function of sine type for $u(\operatorname{Re} z)$. We must prove that the estimates of Theorem 1 are fulfilled outside the disks $B\left(z_{n k}, \varepsilon \tau\left(u, b_{n}\right)\right)$ and that these disks are mutually disjoint. These statements are consequences of the following lemma.

Lemma 3. If $u$ is a convex function on $\mathbb{R}$, then

$$
\rho(u, \operatorname{Re} z) \leq \tau(u, z) \leq 24 \rho(u, \operatorname{Re} z)
$$

for every $z \in \mathbb{C}$.

Proof. The left inequality follows from (2) because $l_{u}(y, \operatorname{Re} z)$ is a harmonic function.

Let $H(w)$ be a function harmonic in the disk $B=B(z, \tau(u, z))$ and satisfying the inequality $|u(\operatorname{Re} w)-H(w)| \leq 1$. We denote by $L(z, x)$ the linear function tangent to $u$ at the point $\operatorname{Re} z$, i.e., $L(z, x) \leq u(x)$ for all $x$ and $L(z, \operatorname{Re} z)=u(\operatorname{Re} z)$.

Then

$$
L(z, \operatorname{Re} w) \leq u(\operatorname{Re} w) \leq H(w)+1
$$

for all $w \in B$, and $L(z, \operatorname{Re} z)=u(\operatorname{Re} z) \geq H(z)-1$. The function $v(w)=H(w)-$ $L(z, \operatorname{Re} w)+1$ is harmonic in $B$ and satisfies $v(w) \geq 0, v(z) \leq 2$. Applying the Harnack inequality to $v$ in the disk $B\left(z, \frac{1}{2} \tau(u, z)\right)$, we obtain $v(w) \leq 6$. Thus, $H(w)-L(z, \operatorname{Re} w) \leq$ 5 in that disk and, therefore, $0 \leq u(\operatorname{Re} w)-L(z, \operatorname{Re} w) \leq 6$. We denote the quantity $\tau(u, z)$ by $\tau$. A geometric argument shows that $0 \leq u(x)-L(z, x) \leq \frac{1}{2}$ on the interval $\left[\operatorname{Re} z-\frac{\tau}{24} ; \operatorname{Re} z+\frac{\tau}{24}\right]$. Consequently,

$$
u\left(y-\frac{\tau}{24}\right)+u\left(y+\frac{\tau}{24}\right)-2 u(y) \leq L\left(z, y-\frac{\tau}{24}\right)+L\left(z, y+\frac{\tau}{24}\right)-2 L(z, y)+1=1 .
$$

So, $\frac{\tau}{24} \leq \rho(u, \operatorname{Re} z)$ or $\tau(u, z) \leq 24 \rho(u, \operatorname{Re} z)$.

This completes the proof of Corollary 1.

Corollary 2. If $u$ has asymptotes at $\pm \infty$, then there exists a sine type function for it. 
Proof. As was noted in the proof of Lemma 2, the piecewise linear function $v$ that approximates $u$ has finitely many break points. By Lemma 1, we obtain a finite sum representation

$$
v(x)=L_{0}(x)+\sum_{n=1}^{N} L_{n}^{+}(x) .
$$

The required sine type function is the finite product

$$
f(z)=e^{L_{0}(z)} \prod_{n=1}^{N} F\left(L_{n}(z)\right) .
$$

However, the function $e^{a z} \sinh b z$ for appropriate $a$ and $b$ is also as required in Corollary 2.

We prove a version of Theorem 1 that is more convenient for applications.

Theorem 2. Let $u$ be a two times continuously differentiable convex function on $\mathbb{R}$ such that there is a positive constant $\beta$ and a function $g$ tending to infinity at $\pm \infty$ such that the following conditions are satisfied.

a) For all $x \in \mathbb{R}$ and all $y \in\left[x-\frac{1}{\sqrt{u^{\prime \prime}(x)}} ; x+\frac{1}{\sqrt{u^{\prime \prime}(x)}}\right]$ we have $|\beta(x)-\beta(y)| \leq b$.

b) The integral

$$
\int_{\mathbb{R}} e^{-\varepsilon \beta(x)} \sqrt{u^{\prime \prime}(x)} d x
$$

converges for every $\varepsilon>0$.

c) For every $x \in \mathbb{R}$ and $y_{1}, y_{2} \in\left[x-\frac{\beta(x)}{\sqrt{u^{\prime \prime}(x)}} ; x+\frac{\beta(x)}{\sqrt{u^{\prime \prime}(x)}}\right]$ we have

$$
\frac{u^{\prime \prime}\left(y_{1}\right)}{u^{\prime \prime}\left(y_{2}\right)} \geq b>0 \text {. }
$$

Then there exists an entire sine type function $u$ that has simple zeros of the form $z_{n k}=$ $b_{n}+\frac{2 \pi k i}{d_{n}}$ and for every $\varepsilon>0$ satisfies the inequality

$$
|\ln | f(z)|-u(z)| \leq A(\varepsilon)
$$

outside of the disks $B\left(z_{n k}, \frac{\varepsilon}{\sqrt{u^{\prime \prime}\left(b_{n}\right)}}\right)$. Moreover, these disks are mutually disjoint if $\varepsilon>0$ is sufficiently small.

Proof. We show that

$$
\sqrt{b} \rho(u, x) \leq \frac{1}{\sqrt{u^{\prime \prime}(x)}} \leq \frac{\rho(u, x)}{\sqrt{b}}
$$

for sufficiently large $|x|$ if the assumptions of the theorem hold true. Take $p>0$. The Taylor formula shows that

$$
u(x+p)+u(x-p)-2 u(x)=\frac{1}{2}\left(u^{\prime \prime}\left(y_{1}\right)+u^{\prime \prime}\left(y_{2}\right)\right) p^{2},
$$

where $\left|y_{j}-x\right| \leq p$. If $p=\sqrt{\frac{b}{u^{\prime \prime}(x)}}$, then assumption c) implies that $u^{\prime \prime}\left(y_{j}\right) \leq \frac{u^{\prime \prime}(x)}{b}$ (clearly, $b \leq \beta(x)$ for large $|x|$ ); consequently,

$$
u(x+p)+u(x-p)-2 u(x) \leq 1 .
$$

This shows that $\rho(u, x) \geq \sqrt{\frac{b}{u^{\prime \prime}(x)}}$. 
We put $p=\sqrt{\frac{1}{b u^{\prime \prime}(x)}}$ in $(10)$. Then

$$
u(x+p)+u(x-p)-2 u(x) \geq 1
$$

whence $\rho(u, x) \leq \sqrt{\frac{1}{b u^{\prime \prime}(x)}}$.

It follows that the function $\alpha(x)=\sqrt{b} \beta(x)$ satisfies the assumptions of Theorem 1 .

As an illustration, we can consider the functions $u(x)=\left(|x|^{2}+1\right)^{p}$, where $p \geq \frac{1}{2}$. Here we take $|x|^{\sigma}(\sigma>0$ is an arbitrary sufficiently small number) for the role of $\beta$.

\section{$\S 2$. Completeness of A system of exponentials in $L^{2}(I, h)$}

Let $h(t)$ be a convex function on a bounded interval $I \subset \mathbb{R}$. We denote by $L^{2}(I, h)$ the space of functions locally integrable on $I$ for which the following integral is finite:

$$
\|f\|^{2}=\int_{I}|f(t)|^{2} e^{-2 h(t)} d t .
$$

Let $\Lambda$ be a subset of the plane. The system of the exponentials $e^{\lambda z}, \lambda \in \Lambda$, will be denoted by $\mathcal{E}(\Lambda)$. For a holomorphic function $L$, we denote by $\Lambda_{L}$ the set of zeros of $L$ (taken without multiplicities).

In this section, we are interested in completeness and minimality of the system $\mathcal{E}\left(\Lambda_{L}\right)$ in $L^{2}(I, h)$ provided $L$ is a sine type function for a convex function $u$.

We need the following theorem from [6].

Theorem A. Let $h(t)$ be a convex function on a bounded interval I, and let $\widetilde{h}(x)=$ $\sup _{t \in I}(x t-h(t))$ be Young conjugate to $h$. Then we have the following statements.

1. For any functional $S$ on $L^{2}(I, h)$, its generalized Laplace transform $\widehat{S}(z)=S\left(e^{z t}\right)$ is an entire function that satisfies the conditions

$$
\begin{aligned}
|\widehat{S}(z)| & \leq C_{S} \exp \widetilde{h}(x), \\
\|\widehat{S}\|^{2} & :=\int_{\mathbb{R}} \int_{\mathbb{R}}|\widehat{S}(x+i y)|^{2} e^{-2 \widetilde{h}(x)} \rho_{\widetilde{h}}(x) d \widetilde{h}^{\prime}(x) d y \leq(\pi e)^{2}\|S\|^{2} ;
\end{aligned}
$$

furthermore, $\|\widehat{S}\| \geq(\pi e)^{-1}\|S\|$.

2. The converse is also true; i.e., if $F$ is an entire function of exponential type satisfying

$$
\begin{gathered}
|F(z)| \leq C_{F} \exp \widetilde{h}(x), \quad z=x+i y, \\
\int_{\mathbb{R}} \int_{\mathbb{R}}|F(x+i y)|^{2} e^{-2 \widetilde{h}(x)} \rho_{\widetilde{h}}(x) \widetilde{d h}^{\prime}(x) d y<\infty,
\end{gathered}
$$

then there is a functional $S \in L^{2}(I, h)^{*}$ with

$$
\widehat{S}(z) \equiv F(z), \quad z \in \mathbb{C} .
$$

We want to prove the following completeness theorem.

Theorem 3. Let $h$ be a convex function on a bounded interval $I$, and let $u$ be a convex function on $\mathbb{R}$. Suppose $L \in \mathcal{S}(u)$. The system $\mathcal{E}\left(\Lambda_{L}\right)$ of exponentials is complete in $L^{2}(I, h)$ if and only if there is no nonlinear convex function $v$ on $\mathbb{R}$ with

$$
v(x)+u(x) \leq \widetilde{h}(x), \quad x \in \mathbb{R} .
$$

Proof. 1. The "if" part. Suppose no convex function with the properties indicated exists, but the system $\mathcal{E}\left(\Lambda_{L}\right)$ is not complete in $L^{2}(I, h)$. By the Banach completeness theorem, there exists a nonzero functional $S$ on $L^{2}(I, h)$ that vanishes on all exponentials

of the system $\mathcal{E}\left(\Lambda_{L}\right)$. The Fourier-Laplace transform $\widehat{S}(z)$ of this functional is an entire 
function that vanishes at every $\lambda \in \Lambda_{L}$ and satisfies the conditions of the first item in Theorem A. Now, $\widehat{S}(z)$ is divisible by $L(z)$; we put $\widehat{S}(z)=g(z) L(z)$. We denote the zeros of $L$ as follows: $\Lambda_{L}=\left\{z_{n}\right\}, n \in \mathbb{N}$. Let $B_{n}=B\left(z_{n}, \varepsilon \tau\left(u, z_{n}\right)\right)$ be mutually disjoint disks outside of which we have

$$
\frac{1}{A(\varepsilon)} e^{u(x)} \leq|L(x+i y)| \leq A(\varepsilon) e^{u(x)} .
$$

By the properties of $\widehat{S}$ and $L$, we have

$$
\ln |g(z)|=\ln |\widehat{S}(z)|-\ln |L(z)| \leq \widetilde{h}(\operatorname{Re} z)-u(\operatorname{Re} z)+C
$$

for $z \notin \cup B_{n}$. It follows that the entire function $g$ is of exponential type; moreover, if $g(z)=\exp (a z)$, then $a$ must be real in view of boundedness on vertical lines outside $\cup B_{n}$. However, if $a$ is real, then the integral in the first item of Theorem A diverges. Thus, $g$ is not an exponential. Much in the same way, we prove that $g$ cannot be of the form $\exp (a z) p(z)$, where $p$ is a polynomial. Thus, $g$ has infinitely many zeros.

Assume that $\varepsilon<1$ in the definition of the disks $B_{n}$. We denote by $H_{n}$ a function harmonic in the disk $B\left(z_{n}, \tau\left(u, z_{n}\right)\right)$ and deviating from $u(z)$ by at most 1 . Such a function exists by the definition of $\tau\left(u, z_{n}\right)$. On the boundary of $B_{n}$, we have

$$
\begin{aligned}
\ln \mid z & -z_{n}|-\ln | L(z) \mid+H_{n}(z) \\
& \leq u(z)-\ln |L(z)|+\ln \left(\varepsilon \tau\left(u, z_{n}\right)\right)+H_{n}(z)-u(z) \\
& \leq A(\varepsilon)+\ln \left(\varepsilon \tau\left(u, z_{n}\right)\right)+1 \leq \mathrm{const}+\ln \tau\left(u, z_{n}\right) .
\end{aligned}
$$

By the maximum principle for harmonic functions, this estimate extends to the interior of the disk; that is, whenever $z \in B_{m}$ for some $m$, we have

$$
\ln \left|z-z_{n}\right|-\ln |L(z)|+H_{n}(z) \leq \mathrm{const}+\ln \tau\left(u, z_{m}\right) .
$$

Now, Lemma 3 and property (1) imply that

$$
\ln \tau\left(u, z_{m}\right) \leq 4+\ln \rho\left(u, \operatorname{Re} z_{m}\right) \leq \mathrm{const}+\ln \left(\left|\operatorname{Re} z_{m}\right|+1\right) .
$$

The last two formulas show that

$$
-\ln |L(z)|+\ln \left|z-z_{n}\right|+u(z) \leq \mathrm{const}+\ln (|\operatorname{Re} z|+1)
$$

for $z \in \bigcup B_{m}$. The Young conjugate function $\widetilde{h}$ satisfies the Lipschitz condition

$$
|\widetilde{h}(x)-\widetilde{h}(y)| \leq \max _{t \in I}|t||x-y|, \quad x, y \in \mathbb{R} .
$$

Fix some $n$. If $\left|z-z_{n}\right| \geq 1$, then

$$
\ln |\widehat{S}(z)|-\ln \left|z-z_{n}\right| \leq \ln |\widehat{S}(z)| \leq \mathrm{const}+\widetilde{h}(\operatorname{Re} z) .
$$

If $\left|z-z_{n}\right| \leq 1$, the maximum principle for subharmonic functions yields

$$
\ln |\widehat{S}(z)|-\ln \left|z-z_{n}\right| \leq \mathrm{const}+\max _{\left|\zeta-z_{n}\right| \leq 1} \widetilde{h}(\operatorname{Re} \zeta)
$$

Together with the Lipschitz property for $\widetilde{h}$, this shows that (13) is valid for all $z$. Now (11), (12), and (13) imply that

$$
\ln |g(z)| \leq \widetilde{h}(\operatorname{Re} z)-u(\operatorname{Re} z)+\mathrm{const}+\ln (|\operatorname{Re} z|+1)
$$

for all $z$. At the beginning of the proof, we observed that $g$ has infinitely many zeros. Let $\zeta_{0}, \zeta_{1}$ be zeros of $g$, and let

$$
g_{0}(z)=\frac{g(z)}{z-\zeta_{0}}, \quad g_{1}(z)=\frac{g_{0}(z)}{z-\zeta_{1}} .
$$


The last estimate for $g$ shows that

$$
\ln \left|g_{0}(z)\right| \leq \widetilde{h}(\operatorname{Re} z)-u(\operatorname{Re} z)+C_{0}, \quad \ln \left|g_{1}(z)\right| \leq \widetilde{h}(\operatorname{Re} z)-u(\operatorname{Re} z)+C_{1} .
$$

Now, the functions

$$
v_{0}(x)=\sup _{y} \ln \left|g_{0}(x+i y)\right|, \quad v_{1}(x)=\sup _{y} \ln \left|g_{1}(x+i y)\right|
$$

are convex, and

$$
v_{0}(x)+u(x) \leq \widetilde{h}(x)+C_{0}, \quad v_{1}(x)+u(x) \leq \widetilde{h}(x)+C_{1} .
$$

If at least one of these functions is nonlinear, we arrive at a contradiction (there are no such functions by assumption). Suppose both $v_{0}$ and $v_{1}$ are linear. If $\left|x-\operatorname{Re} \zeta_{1}\right|>1$, the definition of the $v_{j}$ implies $v_{1}(x)<v_{0}(x)$. Consequently, the slopes of these functions coincide. Let $v_{j}(x)=a x+b_{j}$. Since

$$
\ln \left|g_{1}(z)\right|=\ln \left|g_{0}(z)\right|-\ln \left|z-\zeta_{1}\right| \leq\left|g_{0}(z)\right|-\ln \left|\operatorname{Re}\left(z-\zeta_{1}\right)\right|,
$$

we have

$$
a x+b_{1} \leq a x+b_{0}-\ln \left|x-\operatorname{Re} \zeta_{1}\right|,
$$

or $\ln \left|x-\operatorname{Re} \zeta_{1}\right| \leq b_{0}-b_{1}$. This contradiction shows that at least one of the $v_{j}$ is nonlinear. If, say, $v_{1}$ is nonlinear, we put $v(x)=v_{1}(x)-C_{1}$; then $v(x)+u(x) \leq \widetilde{h}(x)$.

2. The "only if" part. Suppose that $v(x)+u(x) \leq \widetilde{h}(x)$ for some nonlinear convex function $v$. We show that then the system $\mathcal{E}\left(\Lambda_{L}\right)$ is not complete in $L^{2}(I, h)$. Let $l_{1}(x)$, $l_{2}(x)$ be two different linear functions tangent to $v$. The function $l(x)=\max \left(l_{1}(x), l_{2}(x)\right)$ is convex and linear near $\pm \infty$, and $l(x) \leq v(x)$. By the corollary to Theorem 1, there exists a sine type function $g_{0}$ for $L$. Let $\zeta_{1}, \zeta_{2}$ be different zeros of $g_{0}$. Put

$$
g(z)=\frac{g_{0}(z)}{\left(z-\zeta_{1}\right)\left(z-\zeta_{2}\right)} .
$$

Property $2^{\prime}$ of sine type functions shows that

$$
|g(z)| \leq C\left(\zeta_{1}, \zeta_{2}\right) \frac{e^{v(\operatorname{Re} z)}}{(|z|+1)^{2}}, \quad z \in \mathbb{C} .
$$

It can easily be seen that the function $F(z)=g(z) L(z)$ satisfies the conditions of item 2 in Theorem A; consequently, it is the Laplace-Fourier transform of some nonzero functional $S$ on $L^{2}(I, h)$. This functional vanishes at all exponentials of the system $\mathcal{E}\left(\Lambda_{L}\right)$, so this system is not complete by the Banach theorem.

Theorem 4. Let $h$ be a convex function on a bounded interval $I=(a ; b)$, and let $u$ be a convex function on $\mathbb{R}$. Next, let $L \in \mathcal{S}(u)$ be a sine type function for $u$. The system $\mathcal{E}\left(\Lambda_{L}\right)$ of exponentials is complete in $L^{2}(I, h)$ if and only if

$$
\lim _{x \rightarrow-\infty} \frac{u(x)}{|x|}+\lim _{x \rightarrow+\infty} \frac{u(x)}{|x|} \geq b-a .
$$

Proof. The proof will be prefaced with the following lemma.

Lemma 4. 1. Let $v$ be a convex function on $\mathbb{R}$. Then the limits (which are finite or equal to $+\infty$ )

$$
\lim _{x \rightarrow \pm \infty} \frac{v(x)}{|x|}
$$

exist and their sum is nonnegative. Moreover, this sum is equal to zero if and only if $v$ is a linear function. 
2. If $h$ is a convex function on a bounded interval $(a, b)$ and $\widetilde{h}$ is Young conjugate to $h$, then

$$
\lim _{x \rightarrow-\infty} \frac{\widetilde{h}(x)}{|x|}=-a, \quad \lim _{x \rightarrow+\infty} \frac{\widetilde{h}(x)}{|x|}=b .
$$

Proof. We observe that

$$
\liminf _{x \rightarrow+\infty} \frac{v(x)}{x}>-\infty
$$

because a convex function majorizes its tangent lines. Put

$$
\liminf _{x \rightarrow+\infty} \frac{v(x)}{x}=a .
$$

If $a=+\infty$, the claim of the first part is true. Suppose $-\infty<a<+\infty$. Then for every $\varepsilon>0$ we have $v(x) \leq(a+\varepsilon) x$ on some sequence $x_{n}$. By the convexity of $v(x)$, these inequalities are also fulfilled between these points, i.e., for all $x>x_{1}$. Then

$$
\limsup _{x \rightarrow+\infty} \frac{v(x)}{x} \leq a+\varepsilon .
$$

Since $\varepsilon>0$ is arbitrary, we obtain

$$
\limsup _{x \rightarrow+\infty} \frac{v(x)}{x} \leq a,
$$

and, consequently,

$$
\lim _{x \rightarrow+\infty} \frac{v(x)}{x}=a .
$$

The existence of the limit at $-\infty$ is proved similarly. Take any numbers $y_{-}<0, y_{+}>0$. By convexity, we have

$$
v(x) \geq v^{\prime}\left(y_{-}\right)\left(x-y_{-}\right)+v\left(y_{-}\right)
$$

therefore,

$$
\lim _{x \rightarrow-\infty} \frac{v(x)}{|x|} \geq-v^{\prime}\left(y_{-}\right), \quad \lim _{x \rightarrow+\infty} \frac{v(x)}{|x|} \geq v^{\prime}\left(y_{+}\right) .
$$

It follows that

$$
\lim _{x \rightarrow-\infty} \frac{v(x)}{|x|}+\lim _{x \rightarrow+\infty} \frac{v(x)}{|x|} \geq v^{\prime}\left(y_{+}\right)-v^{\prime}\left(y_{-}\right) .
$$

Since $y_{-}<y_{+}$and the derivative of a convex function increases monotonically, we see that $v^{\prime}\left(y_{-}\right) \leq v^{\prime}\left(y_{+}\right)$or $v^{\prime}\left(y_{+}\right)-v^{\prime}\left(y_{-}\right) \geq 0$. Clearly, if $v$ is nonlinear, then $y_{-}, y_{+}$can be chosen in such a way that $v^{\prime}\left(y_{+}\right)-v^{\prime}\left(y_{-}\right)>0$.

The claims of the second part follow from the obvious inequalities (see [8])

$$
\begin{aligned}
&(b-\varepsilon) x-h(b-\varepsilon) \leq \widetilde{h}(x) \leq b x-\min _{t \in(a ; b)} h(t), \quad x>0, \\
&(a+\varepsilon) x-h(a+\varepsilon) \leq \widetilde{h}(x) \leq a x-\min _{t \in(a ; b)} h(t), \quad x<0 .
\end{aligned}
$$

We pass to the proof of Theorem 4 .

1. Suppose the system of exponentials in question is not complete in the space indicated. By Theorem 3, there exists a nonlinear convex function $v$ such that

$$
v(x)+u(x) \leq \widetilde{h}(x) .
$$

By Lemma 4,

$$
\lim _{x \rightarrow+\infty} \frac{v(x)}{|x|}+\lim _{x \rightarrow+\infty} \frac{u(x)}{|x|} \leq b, \quad \lim _{x \rightarrow-\infty} \frac{v(x)}{|x|}+\lim _{x \rightarrow-\infty} \frac{u(x)}{|x|} \leq-a .
$$


Adding these formulas, we arrive at

$$
\lim _{x \rightarrow+\infty} \frac{u(x)}{|x|}+\lim _{x \rightarrow-\infty} \frac{u(x)}{|x|}<b-a .
$$

2. Suppose the last inequality is true. In particular, it shows that the two limits on the left are finite. We put

$$
\lim _{x \rightarrow+\infty} \frac{u(x)}{|x|}=B, \quad \lim _{x \rightarrow-\infty} \frac{u(x)}{|x|}=-A .
$$

Then $B-A<b-a$. Let $B-A=(b-a)-4 \varepsilon$. We set $k=b-B-2 \varepsilon$. Then

$$
\begin{aligned}
& \lim _{x \rightarrow+\infty} \frac{u(x)+k x}{|x|}=b-2 \varepsilon, \\
& \lim _{x \rightarrow-\infty} \frac{u(x)+k x}{|x|}=-A-b+B+2 \varepsilon=-a-2 \varepsilon .
\end{aligned}
$$

Consequently,

$$
\begin{aligned}
& u(x)+k x \leq(b-\varepsilon) x+C_{1}, \quad x>0, \\
& u(x)+k x \leq(a+\varepsilon) x+C_{1}, \quad x<0,
\end{aligned}
$$

with some constant $C_{1}$. By Lemma 4 ,

$$
\begin{aligned}
& \widetilde{h}(x) \geq\left(b-\frac{\varepsilon}{2}\right) x+C_{2}, \quad x>0, \\
& \widetilde{h}(x) \geq\left(a+\frac{\varepsilon}{2}\right) x+C_{2}, \quad x<0,
\end{aligned}
$$

with some constant $C_{2}$. Comparing the estimates obtained for $u(x)+k x$ and $\widetilde{h}(x)$, we deduce that

$$
u(x)+k x \leq \widetilde{h}(x)-\frac{\varepsilon}{2}|x|+C
$$

with some constant $C$. The function

$$
v(x)=\frac{\varepsilon}{2}|x|-C+k x
$$

is nonlinear and convex, and the last inequality implies that $v(x)+u(x) \leq \widetilde{h}(x)$. By Theorem 3 , in this case the system $\mathcal{E}\left(\Lambda_{L}\right)$ is not complete in $L_{2}(I, h)$.

Finally, we prove a minimality theorem for a complete system $\mathcal{E}\left(\Lambda_{L}\right)$ of exponentials constructed by the zeros of a sine type function.

Theorem 5. Let $u$ be a convex function on $\mathbb{R}$, let $L \in \mathcal{S}(u)$, and let $h$ be a convex function on a bounded interval $I$. Then the system $\mathcal{E}\left(\Lambda_{L}\right)$ is minimal in $L^{2}(I, h)$ if and only if

$$
\int \frac{e^{2(u(x)-\widetilde{h}(x))}}{(|x|+1)} \rho(u, x) d \widetilde{h}^{\prime}(x)<\infty, \quad \frac{e^{u(x)}}{|x|+1} \leq C e^{\widetilde{h}(x)} .
$$

Proof. Let $z_{n}$ be the zeros of $L$. The system $\mathcal{E}\left(\Lambda_{L}\right)$ is minimal if and only if the functions $L(z)\left(z-z_{n}\right)^{-1}$ belong to $\widehat{L}^{2}(I, h)$. By Theorem A, for this it is required that

$$
\left|\frac{L(z)}{z-z_{n}}\right| \leq C e^{\widetilde{h}(\operatorname{Re} z)}, \quad \iint\left|\frac{L(z)}{z-z_{n}}\right|^{2} e^{-2 \widetilde{h}(x)} \rho(u, x) d \widetilde{h}^{\prime}(x) d y<\infty .
$$

The claim follows from these conditions and the properties of sine type functions. 


\section{REFERENCES}

[1] B. Ya. Levin and Yu. I. Lyubarskiı̆, Interpolation by entire functions belonging to special classes and related expansions in series of exponentials, Izv. Akad. Nauk SSSR Ser. Mat. 39 (1975), no. 3, 657-702; English transl., Math. USSR-Izv. 9 (1975), no. 3, 621-662. MR0454019 (56:12270)

[2] V.S. Azarin, The rays of completely regular growth of an entire function, Mat. Sb. (N. S.) 79 (121) (1969), no. 4, 463-476; English transl., Math. USSR-Sb. 8 (1969), 437-450. MR0257357 (41:2008)

[3] R. S. Yulmukhametov, Approximation of subharmonic functions, Anal. Math. 11 (1985), no. 3, 257-282. (Russian) MR0822590(88a:31002)

[4] Yu. I. Lyubarskiǐ and M. L. Sodin, Analogues of functions of sinusoidal type for convex domains, Preprint no. 17, Fiz.-Tekhn. Inst. Nizkikh Temperatur Akad. Nauk Ukr. SSR, Khar'kov, 1986. (Russian)

[5] A. F. Leont'ev, Entire functions. Series of exponentials, Nauka, Moscow, 1983. (Russian) MR0753827 (86j:30005)

[6] V. I. Lutsenko and R. S. Yulmukhametov, Generalization of the Paley-Wiener theorem on weighted spaces, Mat. Zametki 48 (1990), no. 5, 80-87; English transl., Math. Notes 48 (1990), no. 5-6, 11311136 (1991). MR:1092157 (92b:30030)

[7] I. E. Chyzhikov, Approximation of subharmonic functions of slow growth, Mat. Fiz. Anal. Geom. 9 (2002), no. 3, 509-520. MR.1949807 (2003i:30057)

[8] K. P. Isaev, A. A. Putintseva, and R. S. Yulmukhametov, Representation by series in weighted spaces on the real axis, Ufim. Mat. Zh. 1 (2009), no. 1, 16-37. (Russian)

[9] B. Ya. Levin, On bases of exponential functions in $L^{2}(-\pi, \pi)$, Zap. Fiz.-Mat. Fak. Khar'kov. Gos. Univ. i Khar'kov. Mat. Obshch. 27 (1961), no. 4, 39-48. (Russian)

[10] Yu. Lyubarskii and E. Malinnikova, On approximation of subharmonic functions, J. Anal. Math. 83 (2001), 121-149. MR1828489 (2002b:30043)

Bashirir State University, Ul. Zaki Validi 32, Ufa 450074, Russia

E-mail address: bashmakov_rustem@mail.ru

Bashkir State University, Ul. Zaki Validi 32, Ufa 450074, Russia

E-mail address: PutinBSU@yandex.ru

Institute of Mathematics with Computer Center, Ul. Chernyshevskogo 112, Ufa 450077, Russia

E-mail address: yulmukhametov@mail.ru

Received 17/JUN/2009

Translated by S. KISLYAKOV 